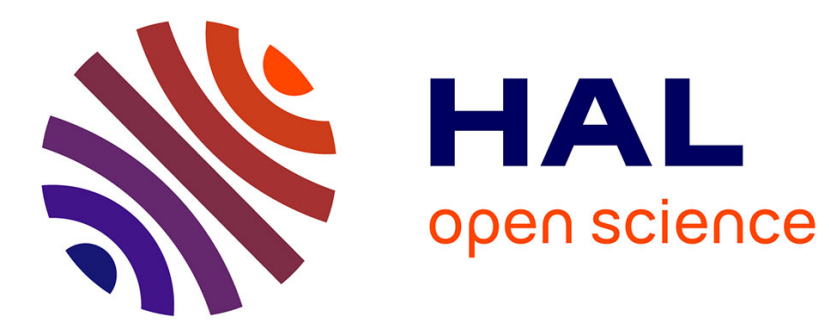

\title{
Les objets des disparus. Exhumations et usages des traces matérielles de la violence de masse
}

\author{
Sophie Baby, François-Xavier Nerard
}

\section{To cite this version:}

Sophie Baby, François-Xavier Nerard. Les objets des disparus. Exhumations et usages des traces matérielles de la violence de masse. Les Cahiers Sirice / Irice , 2017, 19. hal-01705195

\section{HAL Id: hal-01705195 \\ https://u-bourgogne.hal.science/hal-01705195}

Submitted on 9 Apr 2018

HAL is a multi-disciplinary open access archive for the deposit and dissemination of scientific research documents, whether they are published or not. The documents may come from teaching and research institutions in France or abroad, or from public or private research centers.
L'archive ouverte pluridisciplinaire HAL, est destinée au dépôt et à la diffusion de documents scientifiques de niveau recherche, publiés ou non, émanant des établissements d'enseignement et de recherche français ou étrangers, des laboratoires publics ou privés. 


\section{LES OBJETS DES DISPARUS. EXHUMATIONS ET USAGES DES TRACES MATÉRIELLES DE LA VIOLENCE DE MASSE}

Sophie Baby, François-Xavier Nérard

UMR Sirice | «Les Cahiers Sirice»

$2017 / 2 \mathrm{~N}^{\circ} 19$ | pages 5 à 20

ISSN 1967-2713

Article disponible en ligne à l'adresse :

https://www.cairn.info/revue-les-cahiers-sirice-2017-2-page-5.htm

\section{Pour citer cet article :}

Sophie Baby, François-Xavier Nérard « Les objets des disparus. Exhumations et usages des traces matérielles de la violence de masse », Les Cahiers Sirice 2017/2 $\left(\mathrm{N}^{\circ} 19\right)$, p. 5-20.

Distribution électronique Cairn.info pour UMR Sirice.

(C) UMR Sirice. Tous droits réservés pour tous pays.

La reproduction ou représentation de cet article, notamment par photocopie, n'est autorisée que dans les limites des conditions générales d'utilisation du site ou, le cas échéant, des conditions générales de la licence souscrite par votre établissement. Toute autre reproduction ou représentation, en tout ou partie, sous quelque forme et de quelque manière que ce soit, est interdite sauf accord préalable et écrit de l'éditeur, en dehors des cas prévus par la législation en vigueur en France. Il est précisé que son stockage dans une base de données est également interdit. 


\title{
Les objets des disparus
}

\section{Exhumations et usages des traces matérielles de la violence de masse}

\author{
Sophie BABY \& François-Xavier NÉRARD
}

«A boot is more human than a bone »

Layla Renshaw ${ }^{1}$

\begin{abstract}
Dans son dernier film, El botón de nacar, Patricio Guzmán² continue à explorer les ressorts de la mémoire chilienne, qui constitue un fil directeur de sa production artistique. Ici un objet, le bouton de nacre qui donne son titre au film, devient la métaphore d'un sombre passé qui se décline en deux temps, sous la forme de deux histoires croisées. La première est celle de Jemmy Button, un Indien de la Terre de Feu, de l'ethnie Yagan, qui aurait été vendu aux Anglais en 1830 pour être emmené au Royaume-Uni, en échange d'un bouton en nacre. La seconde est celle d'un bouton retrouvé récemment au fond de l'océan dans la baie de Quintero (province de Valparaíso), attaché à un rail de chemin de fer, lors des investigations pour établir les crimes commis par le régime du général Pinochet après le renversement de Salvador Allende en septembre 1973. Ces rails permettaient de faire couler au fond de la mer les corps des "disparus », ces victimes de la dictature jetées à la mer après avoir été le plus souvent torturées. Le rail et le bouton ont été transmis en 2004 par le juge Juan Guzmán au musée de la Villa Grimaldi, à Santiago. La trace matérielle, preuve du crime, est devenue partie intégrante d'une installation, le monument Rieles³, inaugurée en 2007. Un objet, ce bouton de nacre, sert ainsi de métaphore pour dénoncer un double crime, celui commis contre les Indigènes du continent américain
\end{abstract}

1 Témoignage de l'un des dirigeants de l'Asociación para la Recuperación de la Memoria Histórica cité par Layla Renshaw, Exhuming Loss. Memory, Materiality and Mass Graves of the Spanish Civil War, Walnut Creek, California, Left Coast Press, 2011, p. 159.

2 Le film est sorti en France, le 28 octobre 2015, sous le titre Le bouton de nacre.

3 On trouvera des images sur le site du musée de la Villa Grimaldi : http://villagrimaldi.cl/parque-por-la-paz/monumento-rieles-2/, consulté le 31 octobre 2017. 
par les colons européens et celui commis contre les opposants politiques au régime de Pinochet. Dans l'océan résonnent les voix des Indiens de Patagonie tout comme celles des milliers de disparus de la dictature.

C'est précisément à ces objets, traces matérielles de la mort de masse, exhumés au gré du hasard ou de campagnes intentionnelles, qu'est consacré ce numéro des Cahiers Sirice. Comme le suggère la métaphore filée par Guzmán, l'objet est à la fois une source pour la connaissance du passé, la preuve matérielle du massacre et le signe d'une réactivation mémorielle chargée d'émotion dont le destin ultérieur est révélateur de la charge symbolique variable que les sociétés du présent veulent bien lui accorder.

\section{Victimes, fosses communes et le forensic turn: la généalogie d'un projet}

Ce n'est pas un hasard si cette réflexion débute par un exemple sudaméricain. La répression des dictatures chilienne ou argentine s'est traduite par la pratique massive des disparitions: les militants arrêtés étaient engloutis dans les geôles de la dictature pour ne plus jamais réapparaître, tandis que leurs proches étaient abandonnés à leur incertitude quant au sort du prisonnier. Cette pratique est au cœur de la dénonciation de ces régimes menée en premier lieu par les femmes, mères de la place de Mai à Buenos Aires portant un fichu blanc qui évoque le lange de leur enfant disparu ou épouses réduites à danser seules la cueca sola, cette danse traditionnelle chilienne qui se pratique à deux ${ }^{4}$. La disparition pose des questions si spécifiques lorsqu'il s'agit d'établir la vérité ou de dire la justice que la mobilisation des victimes chiliennes, argentines, mais aussi brésiliennes ou uruguayennes a donné lieu à la codification juridique d'un nouveau crime, celui de « disparition forcée », encadré dès 1992 par les Nations unies ${ }^{5}$. Elle rend par ailleurs

4 Antonia Garcia Castro, « Hors-thèmes. La mémoire des survivants et la révolte des ombres : les disparus dans la société chilienne (1973-1995) », Cultures $\mathcal{E}$ Conflits [En ligne], 24-25, hiver 1996-printemps 1997, mis en ligne le 27 mars 2007, consulté le 30 octobre 2017, http:/ / conflits.revues.org.ezproxy.univ-paris1.fr/2163.

5 Déclaration sur la protection de toutes les personnes contre les disparitions forcées, Résolution 47/133, Assemblée générale des Nations unies, adoptée en séance plénière le 18 décembre 1992 :

http://www.ohchr.org/FR/ProfessionalInterest/Pages/ConventionCED.aspx, consulté le 31 octobre 2017. 
d'autant plus impérieuse la nécessité de retrouver des traces, à la fois preuves du crime et signes qui permettent d'entamer le deuil.

Or, on retrouve cet usage massif de la disparition dans les terrains auxquels s'intéresse ce dossier: l'Espagne de la guerre civile de 19361939, l'Union soviétique stalinienne, le Pérou des années 1980 et la Yougoslavie des années 1990. La grande terreur stalinienne emporte ainsi, entre juillet 1937 et novembre 1938, 700000 personnes, sans qu'aucune explication ne soit donnée à leurs proches pour de longues années. En Espagne, on estime à plus de 100000 les personnes encore enfouies dans les 3000 fosses communes du pays. Au Pérou, la commission internationale pour les personnes disparues chiffre à 15000 personnes le nombre de ceux dont on n'a aucune nouvelle au début des années 20106. En Bosnie enfin, ce sont près de 30000 personnes qui ont disparu pendant la guerre.

$C^{\prime}$ est bien pour faire "réapparaître " les personnes disparues" que l'on s'est mis à la recherche des fosses communes. Il a fallu du temps, au moins celui de la fin des régimes auteurs des massacres. Les seules fosses staliniennes fouillées avant la perestroika l'ont été par les nazis lorsqu'ils occupaient l'ouest de l'Union soviétique. En Argentine, il a fallu attendre la chute de la junte militaire pour que soit créée, en 1984, l'Équipe argentine d'anthropologie médico-légale (EAAF) ${ }^{8}$ chargée de mener les fouilles pour retrouver les restes des disparus. Mais le terme des régimes criminels n'entraîne pas, tant s'en faut, la recherche systématique des corps. En Espagne, c'est 25 ans après la mort du général Franco qu'un mouvement significatif d'exhumations a été initié sur l'impulsion de la société civile. Car ces campagnes d'exhumations s'inscrivent aussi dans une dynamique propre à un XXe siècle traversé à la fois par des violences extrêmes d'une ampleur inédite et par l'épanouissement de la figure victimaire.

6 https://www.icmp.int/the-missing/where-are-the-missing/peru/, consulté le 1er novembre 2017.

7 Élisabeth Claverie, «Réapparaître: retrouver les corps des personnes disparues pendant la guerre en Bosnie », Raisons politiques, ${ }^{\circ} 41,2011, \mathrm{p} .14$.

8 On peut consulter leur site: www.eaaf.org, qui comprend notamment un historique de leur action : http://eaaf.typepad.com/founding_of_eaaf/, consulté le 31 octobre 2017. 
La seconde partie du XXe siècle correspond en effet à l'émergence des victimes dans l'espace public. Ce phénomène, désormais bien connu ${ }^{9}$, travaille en profondeur nos sociétés contemporaines. La figure de la victime a supplanté celle du héros dans le récit national qui privilégie une «historiographie des vaincus ${ }^{10}$ où prime le rapport tragique à l'histoire, en même temps que s'est opéré sur le plan anthropologique un basculement de l'économie morale vers la reconnaissance de la souffrance ${ }^{11}$ jusqu'à générer une certaine «culture du traumatisme ${ }^{12}$. La mise en mémoire des «catastrophes ${ }^{13}$ occupe ainsi une place grandissante dans nos sociétés, notamment sous la forme $\mathrm{d}^{\prime}$ aménagement de lieux de mémoire victimaires ${ }^{14}$. Le statut de victime induit désormais des droits à la reconnaissance ${ }^{15}$, à la réparation, à la vérité, à la justice, à tel point que les procédures judiciaires ordinaires elles-mêmes en ont été bouleversées, pour ne plus se centrer exclusivement sur l'accusé, mais mieux prendre en compte la souffrance des victimes, dont on estime qu'elle doit être soulagée au terme du procès ${ }^{16}$. Ladite justice transitionnelle, qui s'impose depuis les années 1990 comme un dogme universel de sortie de conflit, est fondée sur la reconnaissance sociale des victimes et la lutte contre l'impunité. Loin de l'oubli et de l'amnistie qui ont longtemps dominé la logique post-conflit, l'idée s'est imposée qu'il fallait faire face au passé, le

9 Voir Jean-Michel Chaumont, La concurrence des victimes. Génocides, identités, reconnaissance, Paris, La Découverte/Poche, 1997; Guillaume Erner, La société des victimes, Paris, La Découverte, 2006.

10 Reinhart Koselleck, «Mutation de l'expérience et changement de méthode. Esquisse historico-anthropologique », dans L'expérience de l'histoire, Paris, Seuil, 1997, p. 312313

11 Luc Boltanski, La souffrance à distance, Paris, Métailié, 1993 ; Didier Fassin \& Richard Rechtman, L'empire du traumatisme. Enquête sur la condition de victime, Paris, Flammarion, 2007.

12 E. Ann Kaplan, Trauma Culture: The Politics of Terror and Loss in Media and Literature, Rutgers University Press, 2005. Voir aussi J. C. Alexander et alii, Cultural Trauma and Collective Identity, Berkeley, CA, University of California Press, 2001.

13 Henry Rousso, La dernière catastrophe. L'histoire, le présent, le contemporain, Paris, Gallimard, 2012.

14 David El Kenz et François-Xavier Nérard, Commémorer les victimes en Europe, XVIeXXIe siècles, Seyssel, Champ Vallon, 2011.

15 Axel Honneth, La lutte pour la reconnaissance, Paris, Éditions du Cerf, 2000.

16 Voir Éric Wenzel, «Quelle place pour la victime dans l'ancien droit pénal ? », dans Benoît Garnot (dir.), Les victimes, des oubliées de l'histoire?, Rennes, PUR, 2001, p. 19 29 ; Sandrine Lefranc, «La justice de l'après-conflit politique: justice pour les victimes, justice sans tiers ", Négociations, 2, 2015, p. 102. 
connaître, le dire ${ }^{17}$. Les exhumations de fosses sont ainsi devenues indispensables pour satisfaire tout autant le besoin de réparation des victimes que l'exigence de vérité sur le passé et la demande de la justice pénale.

Certes, fouiller des sites d'enfouissement de masse n'est pas une nouveauté pour les archéologues, qui ont toujours vu dans l'archéologie funéraire une source irremplaçable de connaissance du passé. La Première Guerre mondiale avait également constitué une "entreprise sans précédent de rapatriement et d'identification des corps »18 tant en termes de volume qu'en termes d'innovations techniques sur les chantiers de fouille. Pour autant, le dernier quart du XXe siècle correspond à un changement quantitatif et qualitatif majeur qui s'articule autour de cette demande sociale nouvelle de faire réapparaître les morts, mais également de progrès techniques qui autorisent l'implication de la médecine légale et de ses outils (analyse de l'ADN notamment). Le point de départ là encore est en Argentine avec l'équipe de l'EEAF déjà mentionnée. On y retrouve une série de spécialistes venus $\mathrm{du}$ monde entier qui inaugurent de nouvelles pratiques d'exhumation et d'analyse des fosses communes, qui les font circuler et en transmettent l'expérience dans les zones de conflits, jusqu'en Bosnie ou au Rwanda ${ }^{19}$. La guerre yougoslave joue dans ce cadre un rôle central: c'est la première fois que des exhumations massives sont commandées à dessein par le Tribunal pénal international (TPIY) et que celles-ci sont utilisées par la justice pour comprendre les mécanismes des massacres, leur degré d'intentionnalité et de sophistication, et établir l'existence d'un génocide à Srebrenica ${ }^{20}$. Les exhumations de fosses

17 Pierre Hazan, «Les dilemmes de la justice transitionnelle », Mouvements, 53(1), 1, 2008, p. 41-47.

18 Elisabeth Anstett, Jean-Marc Dreyfus, "Why exhume? Why identify?", dans Human Remains and Identification: Mass violence, genocide, and the 'forensic turn', Manchester, Manchester University Press, 2015, p. 2.

19 Dreyfus et Anstett soulignent ainsi le rôle de Clyde Snow que l'on retrouve en Argentine, puis en Bosnie, ibid., p. 4-5. On peut également penser à l'expérience de l'anthropologue médico-légale Clea Koff, dont le livre, surprenant succès de librairie, rend compte des expériences au Rwanda et en ex-Yougoslavie (Bosnie, Croatie, Kosovo) : Clea Koff, The Bone Woman: a Forensic Anthropologist's Search for Truth in the Mass Graves of Rwanda, Bosnia, Croatia, and Kosovo, New York, Random House, 2004, 271 p., traduit en français par Delphine Chevalier, Raymond Clarinard et Isabelle Taudière : Clea Koff, La mémoire des os, Paris, Éditions Héloïse d'Ormesson, 2005, $349 \mathrm{p}$.

20 Élisabeth Claverie, « Réapparaître... », art. cit., p. 25-30. 
communes deviennent dès lors des pratiques plus communes, du Cambodge à l'Afrique du Sud, du Rwanda à l'Espagne ou à l'Irak. L'ampleur du phénomène reste cependant variable : s'il est devenu très important au Rwanda ou en Espagne, il reste ailleurs, en Russie par exemple, encore marginal. Il faut donc se garder de l'illusion d'une pratique massive et généralisée.

La recherche, la fouille et l'examen de fosses communes constituent en effet un processus lourd, qui suppose la mobilisation de nombreux experts (archéologues, anthropologues, historiens, médecins légistes) et le croisement de nombreuses approches et techniques, depuis la quête de l'emplacement de la fosse (à l'aide de témoignages oraux, documents d'archives, photographies aériennes et satellites, archives cadastrales, analyses topographiques du terrain) jusqu'à l'analyse ADN des ossements en laboratoire, en passant par la tenue même du chantier de fouilles (délimitation de la zone, étiquetage des objets ou ossements trouvés, utilisation de détecteurs de métaux, etc. ${ }^{21}$. Au fil des expériences, le processus a été amélioré, encadré et même codifié dans des protocoles scientifiques qui se sont imposés comme les guides d'une exhumation réussie. Ce faisant, il s'est aussi complexifié : ce processus a un coût, en temps, en hommes, en matériel et en argent, qui constitue un véritable frein en l'absence de prise en charge par l'État ou la justice internationale. En Espagne, par exemple, le rythme des exhumations a considérablement diminué après la baisse drastique des subventions publiques à la fin des années 2000. Nombre de fouilles sont alors condamnées à un relatif amateurisme et peuvent sembler artisanales, voire a-scientifiques, comme c'est le cas par exemple bien souvent en Russie.

Cet intérêt renouvelé des sociétés pour les fosses communes et leur exhumation n'a pas manqué, à son tour, d'attirer l'attention des chercheurs. À tel point que certains ont pu parler d'un forensic turn ${ }^{22}$. C'est notamment le cas des chercheurs regroupés autour d'Elisabeth Anstett et Jean-Marc Dreyfus du projet « Corpses of mass violence and genocide », financé par l'European Research Council, mais également en

21 Voir Mike Groen, Nicholas Marquez-Grant et Robe Janaway, Forensic Archaeology: A Global Perspective, Oxford, Wiley Blackwell, 2015, 616 p. et notamment p. 489-498 la contribution de Francisco Etxeberria, Lourdes Herrasti, Fernando Serrulla et Nicholas Marquez-Grant, "Contemporary exhumations in Spain: recovering the missing from the Spanish civil war».

22 Elisabeth Anstett, Jean-Marc Dreyfus, «Why exhume?... », art. cit., p. 4. 
Espagne autour de Francisco Ferrándiz ou encore sur les questions de la Shoah autour de Zuzanna Dziuban ${ }^{23}$. Pour autant ces recherches se concentrent sur la question des restes humains et de leur identification. Le choix de ce numéro est autre: il est de s'intéresser aux objets retrouvés dans ces fosses et donc de décaler notre regard.

En effet, si les fosses communes ${ }^{24}$ abritent les restes humains des victimes, elles contiennent aussi divers objets qui interpellent tout autant les professionnels des exhumations, les chercheurs que les proches des victimes. L'absence d'une réflexion spécifique sur ces objets peut surprendre dans la mesure où ils constituent le cœur des pratiques archéologiques, notamment en archéologie funéraire, et fournissent des éléments à l'histoire de la culture matérielle. Mais cet angle mort est en accord avec les enjeux sociaux du présent qui poussent à focaliser le regard sur les ossements : il s'agit bien avant tout, pour les victimes qui se mobilisent, de retrouver les corps de leurs proches disparus pour dire la vérité sur leur histoire et leur donner une sépulture digne. Trop souvent délaissés et considérés comme des appendices des restes osseux, les objets sont pourtant non seulement riches en informations, mais aussi porteurs d'émotions et d'interrogations multiples. C'est donc à ces objets, signifiants en soi, à leur fonction dans la pratique de l'exhumation et à leurs usages postérieurs dans les pratiques de ré-inhumation et d'entretien du souvenir que ce volume propose de réfléchir.

23 Voir par exemple la série Human remains and violence, qui compte cinq ouvrages publiés : http://www.manchesteruniversitypress.co.uk/series/human-remains-andviolence/ et la revue qui lui est associée (cinq numéros publiés). Voir aussi Sévane Garibian, Élisabeth Anstett et Jean-Marc Dreyfus (dir.), Restos humanos e identificación. Violencia de masa, genocidio y el 'giro forense', Buenos Aires, Mino y Davila, 2017. En Espagne, les ouvrages et projets de Francisco Ferrándiz: El pasado bajo tierra. Exhumaciones contemporáneas de la Guerra Civil, Madrid, Anthropos, 2014 (cf. leur projet H2020 Unrest: http://www.unrest.eu/home/). Ou plus général : Francisco Ferrandiz et Antonius Robben, Necropolitics. Mass Graves and Exhumations in the Age of Human Rights, Philadelphia, University of Pennsylvania Press, 2013. Sur l'Amérique latine : A-M. Losonczy \& V. Robin Azevedo (dir.), 2016, Retour des corps, parcours des âmes. Exhumations et deuil collectif dans le monde hispanique, Paris, Pétra éditions, 2016. Un exemple sur le Guatemala: Victoria Sanford, Buried Secrets: Truth and Human Rigths in Guatemala. New-York, Palgrave MacMillan, 2003. Z. Dziuban (ed.), Mapping the 'Forensic Turn': The Engagements with Materialities of Mass Death in Holocaust Studies and Beyond, Vienne, New Academic Press, 2017.

24 Notre réflexion porte d'abord sur la mort provoquée par l'homme, et donc exclut les morts d'épidémie. Elle se focalise sur les victimes civiles de conflits et/ou de génocide. Même si la question des guerres est toujours présente, elle ne sera donc pas abordée ici. 
Notre travail a l'ambition de la comparaison et de la pluridisciplinarité. Les exemples traités sont divers, centrés sur deux espaces principaux, l'Europe et l'Amérique latine, qui se situent au cœur des renouvellements tant pratiques que théoriques explorés ci-dessus. Par ailleurs, si les auteurs de cette introduction sont historiens, nous avons cherché à rapprocher des spécialistes de plusieurs disciplines qui ne dialoguent pas toujours entre eux. Ce numéro des Cahiers Sirice fait ainsi se confronter les approches de praticiens des chantiers de fouille nécessaires aux ouvertures des fosses et du traitement des données qu'elles fournissent, avec la réflexion historienne sur les usages symboliques, politiques et sociaux portés par cette pratique. Les textes ici rassemblés proviennent donc d'archéologues, d'anthropologues, $\mathrm{d}^{\prime}$ historiens et $\mathrm{d}^{\prime}$ historiens de l'art. On retrouvera cette pluridisciplinarité aussi bien dans la manière de voir que d'écrire. Ces articles pourront surprendre et inciter à penser le spécialiste de l'autre discipline. C'est en tout cas le pari que nous avons fait.

Deux axes forts ont émergé de nos réflexions collectives, organisés autour de ce qu'on pourrait appeler les deux vies de l'objet: avant l'inhumation et après l'exhumation. L'objet est ainsi une source, un lien avec le monde qui précède les fosses communes, celui des victimes tout comme celui des acteurs du crime. Puis une deuxième vie débute pour les objets exhumés: que doit-on alors en faire? Que deviennent-ils? Quels en sont les usages en tant que traces de la mort de masse ?

\section{L'objet-source}

L'objet retrouvé dans les fosses communes est d'abord une trace matérielle de la violence de masse qui a été à l'origine de son enfouissement. À ce titre, il est un support majeur de connaissance, un élément matériel mis à profit par l'expertise archéologique, anthropologique ou médico-légale. Si l'objet ne présente pas toujours la même utilité suivant son état de conservation, qui varie en fonction du temps écoulé entre l'inhumation et l'exhumation ou de la nature des sols, les objets retrouvés dans les cas qui nous occupent ici sont généralement en assez bon état pour fournir de précieuses informations que les experts savent de mieux en mieux extraire.

Le premier objectif des exhumations est d'identifier les restes des personnes enfouies jusqu'à leur donner, dans la mesure du possible, un 
nom. Associé aux documents d'archives, aux témoignages des survivants, aux résultats des analyses archéologiques ou des tests ADN, l'objet est un élément clef du processus d'identification. Parfois, l'objet parle de lui-même, à l'instar des plaques nominatives de soldats ou de ces documents d'identité retrouvés dans des bouteilles placées aux côtés des corps, comme dans l'exceptionnel cimetière de Pampelune en Espagne (Jiménez \& Herrasti). Vêtements, chaussures, bijoux, montres ou lunettes peuvent être reconnus par un membre de la famille de la victime, au point qu'en Yougoslavie (Jugo) comme au Pérou (Saona) des livres de photos de vêtements ont été confectionnés et montrés aux proches dans l'espoir qu'ils puissent reconnaître l'un d'entre eux. En leur absence, un uniforme, une botte de soldat, des pièces de monnaie, des boutons siglés peuvent contribuer, si ce n'est à fournir un nom, une identité individuelle à chacun des corps enfouis, du moins à éclaircir $l^{\prime}$ origine et la trajectoire du groupe d'individus inhumés, par exemple leur origine géographique voire leur nationalité (Shapoval), leur régiment d'appartenance s'ils étaient soldats, leur origine sociale, tandis qu'un kit de rasage ou une épingle à chignon renseignent sur le genre de la victime.

Par-delà l'identification individuelle ou collective, l'objet, en tant qu'élément de la culture matérielle, participe à la reconstitution de l'histoire des victimes de leur vivant et de l'histoire même du massacre. L'objet nous renseigne sur la condition sociale de la victime, sur sa profession (outils), sur ses croyances et ses peurs (croix, chapelets, amulettes), sur ses habitudes (pipes, matériel à rouler des cigarettes, crayons), sur son mode de vie (peignes, épingles à chignon, rasoirs, lunettes, miroirs). L'objet peut ainsi révéler des pratiques mal connues, notamment les nombreuses adaptations du quotidien aux situations de conflits (uniformes trafiqués pour mieux répondre aux réalités du terrain ; manteaux ou chaussures volés à l'ennemi). Curieusement, alors que depuis toujours les archéologues utilisent en ce sens les traces matérielles trouvées sur leur chantier de fouille pour écrire l'histoire sociale et culturelle des femmes et des hommes qui les y ont laissées, les exhumations contemporaines des fosses communes causées par des massacres de masse ne s'intéressent pas, ou très secondairement, à cette dimension de l'objet. Les articles rassemblés ici n'y font pas exception. Les rapports de fouille visent avant tout à identifier les corps et à expliciter la présence de la fosse tout comme les conditions de la mise à mort: les enjeux mémoriels des sociétés du présent priment largement sur les enjeux de la recherche historique. 
À l'inverse, l'histoire du massacre intéresse donc vivement les acteurs à l'œuvre dans les processus d'exhumation. Les objets personnels déjà évoqués peuvent renseigner sur la nature du groupe visé (femmes, hommes, civils, militaires, paysans, citadins, etc.), sur le moment de la mort (montres arrêtées à la même heure) et sur les modalités d'exécution. On trouve en effet dans les fosses nombre d'objets qui ont directement trait à la violence donnée et subie : des menottes, des cordes ou des tissus pour nouer les poignets; des foulards pour bander les yeux des condamnés; des balles et des douilles issues des armes qui ont donné la mort. Ce faisant, l'objet exhumé n'est pas seulement une source d'information sur les victimes, mais aussi sur les intentions et les pratiques des bourreaux. Ainsi, dater le moment de la mort tout comme celui de l'exhumation est considéré comme essentiel, pour comprendre par exemple si les condamnés étaient exécutés à même la fosse, si les cadavres étaient transportés depuis d'autres lieux d'exécutions, voire s'ils étaient transférés de fosse en fosse. En Russie, l'absence de douilles témoigne d'une pratique propre à la Grande Terreur, quand les prisonniers étaient tués dans les centres d'incarcération avant d'être transportés dans des fosses creusées plus loin (Nérard). En Bosnie, les perpétrateurs ont veillé à brouiller les pistes en créant des fosses secondaires, voire tertiaires, dans lesquels étaient enterrés à dessein des corps et des objets exhumés d'autres fosses (Jugo).

En outre, les munitions retrouvées ainsi que les pratiques d'exécution qui se dessinent contribuent à l'identification des auteurs du massacre, celle-ci étant cruciale pour attribuer les responsabilités et lutter contre les comportements négationnistes dans les cas les plus controversés, où les responsabilités ne sont pas clairement établies et sont l'objet d'enjeux politiques et sociaux extrêmement vifs. En Bosnie, mais aussi au Pérou ou dans l'espace de l'ex-Union soviétique, ces procédures d'identification sont centrales à l'heure de construire un récit historique partagé et d'entretenir la mémoire du massacre. L'objet, source de connaissance, peut alors devenir une preuve susceptible d'être utilisée dans une procédure judiciaire. Scène de crime, la fosse contient des indices qui peuvent être cruciaux à l'heure de déterminer les responsabilités historiques et pénales. Cela fut le cas en Bosnie, où des objets issus des fosses servirent d'éléments à charge aux procès du TPIY.

Pourtant, comme tout document source ou preuve, l'objet peut être trompeur, voire volontairement falsifié : seul, il ne peut suffire et peut conduire à l'erreur. Un vêtement a pu être porté successivement par 
plusieurs individus, des chaussures ont pu être volées sur des cadavres enterrés ailleurs, même des matricules de soldats ont pu être échangées. De plus, comme en Bosnie ou en Ukraine, un objet a pu être enterré à dessein pour brouiller la recherche à venir (Jugo). C'est pourquoi les protocoles scientifiques établis au gré des expériences invitent à la prudence et exigent de confronter l'objet aux autres sources contenues dans la fosse ou situées à l'extérieur, dans les archives ou recueillies auprès des familles ou témoins potentiels.

\section{L'objet-signe}

Source et preuve pour les besoins de l'identification et de l'enquête, qui nous informe sur sa première vie en tant qu'objet appartenant à l'un des protagonistes de la scène de crime, l'objet acquiert bien d'autres significations dès lors qu'on s'interroge sur son devenir après l'exhumation. Que devient l'objet une fois sorti de terre ? À qui revientil, en droit et en pratique? De quelle seconde vie bénéficie-t-il après la mort de son précédent acquéreur?

Après avoir servi en tant que source ou preuve, après avoir été examiné, photographié, temporairement mis sous scellé, l'objet peut banalement être détruit: sa fonction première ayant été accomplie, l'objet perd ainsi toute utilité et disparaît. S'il n'est pas détruit, par scrupule scientifique, judiciaire ou éthique, l'objet est relégué aux oubliettes, stocké sous plastique dans les rangées innombrables des instituts d'expertise médico-légale ou dans les sous-sols des cours de Justice, aux côtés des restes osseux qui n'ont pas été réclamés et dont on ne sait parfois que faire, quand il n'est pas tout bonnement ré-enterré sur le lieu de la fosse, comme dans ce fut le cas en Ukraine, sur le site de Bykovnia (Shapoval), où les objets exhumés au cours des différentes campagnes ont été regroupés dans des boîtes et ré-inhumés au même endroit aux côtés d'autres boîtes contenant les restes humains. La destruction, la ré-inhumation collective ou le stockage semblent être le devenir de la plupart de ces objets.

Mais parfois, l'objet possède une autre utilité, terriblement pragmatique et trop souvent négligé par son aspect immoral : il peut tout simplement être vendu pour sa valeur marchande, après avoir été volé ou récupéré légalement par les membres de la famille. C'est le cas des bijoux en or ou, plus étonnant mais finalement très banal, des dents en or 
ôtées des mâchoires des victimes. L'histoire des objets volés sur les corps vivants ou morts des victimes de la Shoah a depuis longtemps suscité l'intérêt des chercheurs : là, c'est précisément leur rareté qui en dit long sur la spoliation nazie qui veillait à mettre à profit tout élément corporel (tels les cheveux) et matériel (vêtements, chaussures, prothèses dentaires, etc.) des victimes qui étaient utilisés à des fins industrielles ou commerciales. Nombre d'objets étaient volés et revendus au marché noir par les civils des environs : c'est ainsi que Regina, dans un témoignage glaçant au Musée-Mémorial de l'Holocauste de Washington, raconte l'histoire de sa prothèse en or qu'elle a encore dans sa bouche et montre poliment à la caméra (Klinger). Tout comme cette paysanne andine qui voulait récupérer la prothèse en or de sa mère assassinée pour couvrir les frais d'enterrement et espérer en retirer une petite somme d'argent, en guise d'héritage post-mortem (Delacroix). L'objet garde ainsi, au-delà de la mort de son précédent propriétaire, sa fonction utilitaire.

Pourtant, comme l'a souligné un activiste espagnol dans la formule reprise en exergue de cette introduction, "une botte est plus humaine qu'un os » 25 . Un os est un os, un crâne est un crâne, tandis qu'un objet est unique et reconnaissable : dès lors, l'objet devient signe, signe de l'être disparu, signe de l'être aimé, signe de l'être recherché, ou tout bonnement signe de l'existence d'un être humain. L'objet, métonymie de la femme ou de l'homme qui le possédait, a ainsi une puissance évocatrice et émotionnelle majeure. Nombreux sont ceux, non seulement du côté des familles mais aussi des acteurs de l'exhumation archéologues, anthropologues, bénévoles, journalistes -, qui racontent avoir été saisis par l'émotion à la vue d'une paire de lunettes cassée, d'un peigne ou d'un kit de couture, alors que leur regard n'est plus aussi aiguisé par les crânes ou les tibias qu'ils sont habitués à découvrir par dizaines. Mettre au jour un objet personnel est comme une intrusion soudaine dans l'intimité d'une vie dont on se prend à imaginer des fragments, un mariage à la vue d'un anneau, une scène banale de rasage à la vue d'une lame et d'un miroir, la coquetterie d'une femme aux cheveux longs à la vue d'épingles raffinées, l'innocence d'un enfant arraché à son devenir à la vue d'une petite chaussure ornée d'un pompon, ou l'espoir - qui transparaît à la vue d'une amulette portée autour du cou -, d'un soldat d'être protégé des dangers de la guerre. L'objet redonne chair aux os inanimés et l'insertion dans cette banalité

25 Témoignage de l'un des dirigeants de l'Asociación para la Recuperación de la Memoria Histórica cité par Layla Renshaw, Exhuming Loss, op. cit. 
du quotidien participe à la compréhension sensible du surgissement de l'inouï dans la vie d'un être humain. C'est précisément cette puissance émotionnelle de l'objet qui explique qu'une seconde vie puisse lui être accordée jusqu'à devenir, selon les termes employés plus loin par Admir Jugo, une « relique».

Généralement, quand l'exhumation permet aux familles de retrouver le corps d'un proche, comme c'est le cas en Espagne ou au Pérou, les restes osseux tout comme les objets personnels leur sont restitués. Ces derniers retournent ainsi dans la sphère intime qu'ils n'auraient jamais dû quitter, participant au travail de deuil qui n'avait pu être accompli dans sa plénitude. La disparition du corps de l'être cher, en effet, a cela de particulier qu'elle maintient indéfiniment ses proches dans l'incertitude et, par l'absence des rituels funéraires qu'elle induit, empêche le deuil de s'opérer. Dans le cas des sociétés indiennes des plateaux des Andes, comme le montre Dorothée Delacroix, le défunt devient même une âme errante en perdition qui hante jusqu'aux rêves de sa veuve. Le vêtement retrouvé, même souillé de sang, sert alors de support au passage du mort dans l'autre monde, au travers de pratiques rituelles réinventées pour répondre à ces situations exceptionnelles. Que l'objet soit ré-inhumé avec le défunt pour des raisons rituelles, qu'il rejoigne l'intimité familiale en trônant fièrement sur la cheminée du salon aux côtés d'une photo du disparu ou, à l'inverse, en rejoignant d'autres vieilles boîtes à souvenirs au fond d'un placard, il joue un rôle essentiel dans le processus de deuil et l'entretien du souvenir.

C'est cette même puissance évocatrice qui explique que l'objet soit devenu un élément clef des espaces muséographiques dédiés à l'histoire et au souvenir des violences de masse. Si certains mémoriaux choisissent d'exposer des crânes et des ossements, comme c'est le cas au Rwanda, la plupart en Occident préfèrent exposer des documents d'archives, des photographies, des témoignages et des objets ${ }^{26}$. L'objet retrouvé sur une scène de massacre et l'objet exhumé de fosses communes prennent une dimension collective et une signification universelle tout en gardant leur pouvoir d'individualisation de la victime : le visiteur créé par l'objet un

26 C'est notamment le cas au mémorial du 9/11 à New York, dont les collections rassemblent plus de 11000 objets. Les services du musée ont publié un livre rassemblant des photographies des objets retrouvés sur le site de Ground Zero ou donnés par les parents des victimes. Michael Bloomberg, Clifford Chanin, Alice M. Greenwald et Joe Daniels, The stories they tell: artifacts from the National September 11 Memorial Museum : a journey of remembrance, New York, Skira Rizzoli, 2013, 160 p. 
lien sensible avec la victime du temps de son vivant et appréhende ainsi autrement la tragédie. Jane Klinger parle de "survivor object », d'objet survivant, trace matérielle du crime. Une trace qui prend un relief d'autant plus signifiant en l'absence de traces, dans le cas de la Shoah notamment, où chaque objet acquiert une valeur historique et testimoniale d'envergure. L' «objet survivant», quand il désigne ces objets cachés volontairement par les condamnés dans un acte ultime de défi et de liberté sous la terre des camps d'extermination ou à proximité des fosses, prend alors une dimension universelle, voire sacrée, qui justifie d'autant plus l'emploi de la notion de « relique ».

En outre, et c'est ce qui a intéressé dans ce numéro tant Margarita Saona que Stephenie Young, les artistes se sont à leur tour emparés de ces objets pour en faire le cœur d'un art engagé qui s'inscrit dans les processus collectifs de mémoire à l'œuvre au Pérou ou en Bosnie. Loin d'être un objet-relique, l'objet est alors transformé et revivifié. La notion de forensic imagination, d'imagination médico-légale, développée par Stephenie Young met l'accent sur les bouleversements provoqués sur les imaginaires de mémoires par la pratique croissante des exhumations scientifiques de fosses au point que les arts s'en emparent à leur tour, créant des espaces propices à la prise de conscience, mais aussi à la contemplation et à la réflexion, par-delà les seuls espaces des tribunaux et les laboratoires.

La photographie de l'objet exhumé, réalisée par les experts de l'exhumation eux-mêmes, mais aussi par des photographes professionnels convoqués à dessein pour médiatiser l'événement ou présents sur les lieux à titre individuel, est souvent utilisée dans les entreprises de diffusion à des fins journalistiques, militantes ou pédagogiques. Plus encore en cas de disparition ou de détérioration des objets trouvés dans les fosses, leur image photographiée peut servir de preuve à l'appui des procédures judiciaires ou d'indice pour aider à l'identification des victimes, comme dans le cas de ces livres de vêtements qui sont en fait des livres de photographies de vêtements présentés aux familles. C'est alors l'image de l'objet qui devient à son tour un objet, une preuve. Les artistes bosniaques partent ainsi en quête de leurs propres preuves, matériaux trouvés sur les lieux des crimes, pris en photo ou filmés et mis ensuite à la disposition d'un large public. Ces living memorials, ces mémoriaux vivants se retrouvent sur le web ou dans des musées, comme pour l'artiste Sejla Kamerić qui a réalisé une installation sous la forme d'un immense frigidaire, semblable à ceux que 
l'on trouve dans les laboratoires, rempli non pas d'ossements, mais de preuves matérielles médico-légales (Young). Les œuvres des artistes péruviens présentés par Margarita Saona, par le travail sur la matérialité, la texture, la fibre même du tissu, ou la cavité d'un trou de projectile sur un crâne ou un vêtement proposent une expérience esthétique et sensible des traces de la violence. Tout comme l'utilisation des photographies des victimes est une pratique répandue parmi les artistes contemporains, en ce qu'elles figurent de la façon la plus immédiate l'image de l'absent, la photographie et l'image filmée des objets acquièrent elles aussi une dimension esthétique qui prolonge autrement dans l'espace social l'imaginaire des massacres autour de la scène qu'est la fosse commune.

Les huit articles rassemblés ici instruisent, chacun à leur façon, ce dossier. Un premier ensemble étudie les traces matérielles de trois conflits (guerre en ex-Yougoslavie, guerre d'Espagne et terreur stalinienne) et fait dialoguer un anthropologue (Admir Jugo), des archéologues (Jimi Jimenez et Lourdes Herrasti) et un historien (Yuri Shapoval) en se situant sur la longue durée des exhumations: les premières ont lieu à Bykovnia, près de Kiev, en 1941, les plus récentes dans les années 2000 en Espagne. Un deuxième groupe interroge les pratiques d'exhumation d'objets et leur sens. Le contexte chronologique de l'exhumation est ainsi souligné par François-Xavier Nérard (fosses communes du stalinisme) tandis que Jane Klinger réfléchit au peu de traces laissées par la Shoah et à l'importance particulière des objets retrouvés. Dorothée Delacroix, anthropologue, travaille quant à elle sur la réutilisation des objets exhumés des fosses communes péruviennes et au sens qu'il faut lui donner. C'est précisément cette réappropriation des objets exhumés par des artistes contemporains que les derniers articles de ce numéro abordent. Margarita Saona étudie, elle aussi, le terrain péruvien tandis que Stephenie Young conclut ce volume avec l'exYougoslavie.

En refermant cette introduction ${ }^{27}$, nous ne pouvons pas ne pas penser au drame terrible qui se déroule sur la Méditerranée où des milliers de

27 Ce numéro s'inscrit dans la continuité d'un colloque international organisé à Marseille en novembre 2015: il est le fruit des échanges intenses entre tous ses participants, nous leurs en sommes ici reconnaissants. Le projet a été soutenu par 
réfugiés meurent chaque semaine, transformant cette mer en une immense fosse commune, emplie d'épaves, de cadavres et d'objets abandonnés par ces naufragés désespérés. Ces restes, traces matérielles d'une tragédie qui se déroule au temps présent, seront-ils à leur tour un jour retrouvés, exhumés et analysés pour que leur histoire soit enfin écrite et leur dignité retrouvée?
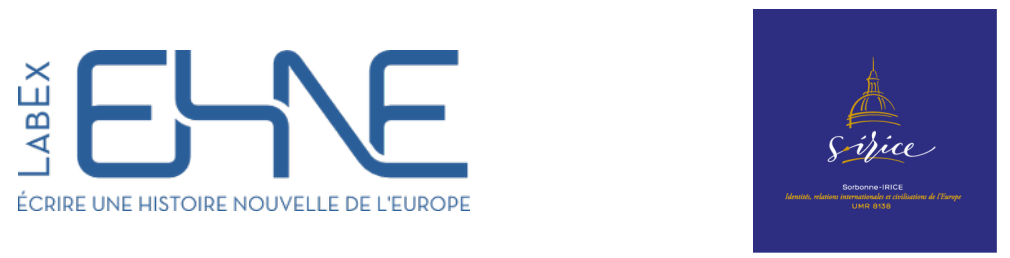

différentes structures et organisations que nous souhaitons remercier: nos universités, nos laboratoires de recherche (le Centre George Chevrier à Dijon et l'UMR Sirice à Paris), mais également le laboratoire ADES à Marseille, dirigé par Michel Signoli qui nous a généreusement accueilli. Le soutien, enfin, de la Région Provence-Alpes-Côte-d'Azur, du ministère de l'Enseignement supérieur et de la Fondation pour la mémoire de la Shoah ont été plus que précieux. Le LabEx EHNE (Écrire une nouvelle histoire de l'Europe) a soutenu depuis le début ce projet facilitant aussi bien l'organisation du colloque que la publication de ce volume. 